\title{
High diagnostic stability of confirmed migraine and confirmed tension-type headache according to the ICHD-3 beta in adolescents
}

\author{
Lucia Albers $^{1 *}$, Andreas Straube ${ }^{2}$, Mirjam N Landgraf ${ }^{3}$, Florian Heinen ${ }^{3}$ and Rüdiger von Kries ${ }^{1}$
}

\begin{abstract}
Background: Stable headache diagnosis classification is a prerequisite for identification of headache type specific risk factors. Does the stability of a headache diagnosis over time vary between migraine and tension-type headache $(\mathrm{TTH})$ ? Are there differences in diagnosis stability between a probable and a definite headache diagnosis?

Findings: In a sample of 783 students (ages 12 to 18 years) participating in a headache intervention study in greater Munich, the stability of headache classification according to the International Classification of Headache Disorder - third edition (beta version) (ICHD-3 beta) after a follow-up of 7 months was examined. Differences in stability of probable or definite migraine and probable or definite $\mathrm{TTH}$ were assessed. The stability of the headache diagnosis was assessed as predictive value of headache diagnosis with regard to confirmation of the headache type using the same diagnostic instrument 7 months later. Predictive values with 95\% confidence intervals (Cl) are reported.

Of students with initial migraine, a diagnosis of migraine was confirmed in $65.71 \%$ of students after 7 months (95\%-Cl [59.40-71.64]). A clear distinction between probable (44.71\%, 95\%-Cl [33.91-53.89]) and confirmed diagnosis (76.88\% 95\%-Cl [69.56-83.17]) of migraine was observed. For TTH the predictive value was $62.66 \%$ (95\%-Cl [57.07-68.01]) overall with a lower stability for probable (46.10\%, 95\%-Cl [37.68-54.69]) compared to the confirmed diagnosis (69.71\%, 95\%-Cl [23.58-37.67]).
\end{abstract}

Conclusion: While confirmed migraine and confirmed TTH diagnoses seem stable over time, stability of a probable diagnosis for either headache type was lower.

Trial registration: The trial was registered at the German Clinical Trial Register with the ID DRKS00003308.

Keywords: Headache; Migraine; Tension-type headache; Adolescents; Stability; Tracking; Headache type; Probable diagnosis; Confirmed diagnosis

\section{Introduction}

Headache is a common health complaint in children and adolescents. A recent review reporting on 64 cross-sectional studies from the last 25 years from 32 different countries and including a total of 227,249 children and adolescents estimated an overall mean prevalence of headache of $54.4 \%$ (95\%-CI $[43.1 ; 65.8])$ and an overall mean prevalence of migraine of 9.1\% (95\%-CI [7.1-11.1]) [1]. Correct classification of the headache type is a prerequisite for targeted treatment

\footnotetext{
* Correspondence: lucia.albers@med.uni-muenchen.de

'Division of Epidemiology, Institute of Social Paediatrics and Adolescent Medicine, Ludwig-Maximilians-University Munich, Haydnstr. 5, 80337 Munich, Germany

Full list of author information is available at the end of the article
}

[2]. In face of the high prevalence of headache in children and adolescents, prevention of headache is a major public health challenge. Epidemiological studies are required for the identification of appropriate preventive interventions. In these studies classification of headache types often cannot be based on an assessment by a physician. While headache in general appears to be a stable trait over time [3], some fluctuation regarding the type of headache has been reported in clinical cohorts [4-6], where headache classification was based on physicians' diagnoses, as well as in epidemiological cohorts, where classification was based on questionnaires according to the International Classification of Headache Disorder [7-10]. 
Potential causes for these fluctuations in reported headache types are: A) Presence of genuinely two different types of headache in a person with different prevailing symptoms over time, of which only the dominant symptoms determine diagnostic classification. B) Genuine Change of headache type over time. C) Poor reliability of the questionnaire or the physicians' diagnosis. D) Poor validity of the questionnaire. In the setting of a cluster randomised trial, we had the opportunity to assess diagnostic stability of probable and confirmed TTH and migraine diagnoses over a seven-month period: How stable is the ICHD classification of Migraine and TTH? Does the stability vary differently between confirmed and probable migraine or TTH?

\section{Findings}

\section{Population}

Our study population consists of 783 students recruited for the headache intervention study MUKIS (acronym for Münchner Untersuchung zu Kopfschmerzen bei Gymnasiasten - Interventionsstudie). MUKIS is a two-armed, prospective intervention study consisting of a baseline inquiry followed by an hour long in-class headache prevention lesson focusing on preventable risk factors for headache, as well as a follow-up inquiry approximately 7 months after the intervention. 12 grammar schools participated in the study. Participants were restricted to students of the 8th, 9th and 10th grades. Inclusion criteria for the present analysis was presence of headache (in the preceding 6 months) both at baseline and at follow-up $(\mathrm{N}=783)$.

The mean age of the included students was 14.4 (range: 12-18 years) with a slight predominance of girls $(62 \%, \mathrm{~N}=489)$.

The study was approved by the Data Safety Officer and the Ethics Committee of the Medical Faculty of the Ludwig-Maximilians-University Munich and the Bavarian Ministry for Teaching and Culture. Parents and students ( $>14$ years of age) gave written consent to participate in the study.

\section{Assessment of headache and clinical characteristics}

Headache characteristics and headache type were assessed using a validated pain questionnaire for children and adolescents. Specific questions were added to the questionnaire to further classify headache subtype as migraine or tension-type headache (TTH) according the classification of the International Headache Society [11]. These additional questions provided all items necessary for diagnosis of TTH and migraine (both probable and confirmed diagnosis) according to the ICHD-3 beta version [12]. Frequency (days with headache) and intensity (on a 10 point visual analogue scale: mild headache intensity $1-3$, moderate headache intensity 4-7 and severe headache intensity
7-10) in the preceding three months was assessed. For the classification of migraine, a headache duration of 4$72 \mathrm{~h}$ was required. Subjects fulfilling the criteria for confirmed migraine or confirmed TTH were labeled as such irrespective of potential symptoms for probable migraine or probable TTH. Individuals with both probable migraine and probable TTH criteria were given a combined diagnosis of migraine plus TTH. All subjects with headache that did not match any of these classifications for primary headache were considered to have miscellaneous headache (MiscH).

\section{Statistical analysis}

The stability of headache diagnosis was examined by evaluating how well the initial headache diagnosis predicted the headache type seven months later, using the same diagnostic instrument. The frequencies of shifting classification of initial headache type at baseline to follow-up were calculated. Differences in stability between probable or definite migraine respectively TTH were assessed using Chi-Square statistics. A stable diagnosis of either migraine or TTH was assumed if adolescents with migraine at baseline had migraine or migraine plus TTH at follow-up, and respectively if adolescents with TTH at baseline had TTH or migraine plus TTH at follow-up.

\section{Study results}

Diagnostic classification of headache did not change significantly for any of the categories in relation to the intervention (Migraine: $\mathrm{p}=0.53$, TTH: $\mathrm{p}=0.97$, Migraine $+\mathrm{TTH}$ : $\mathrm{p}=0.45$, MiscH: $\mathrm{p}=0.55$ ). Therefore merging the intervention and control groups to assess the stability of headache classification over time was justifiable.

The overall stability of classification of migraine and TTH were both moderate (Table 1): On average, 60\% of the adolescents with persistent headache classified as migraine or TTH had TTH or migraine or a combined diagnosis at follow-up.

Considerable proportions of the initial TTH and migraine diagnoses were probable: $44.62 \%$ for TTH and $34.69 \%$ for migraine (Figures 1 and 2). The stability of an initially confirmed TTH diagnosis $(\mathrm{N}=175)$ was clearly better than for an initially probable TTH $(\mathrm{N}=141)$ (Figure 1$)$. In migraine these differences were even more pronounced $(\mathrm{p}<0.001)$ : $76.88 \%$ adolescents with a confirmed migraine initially $(\mathrm{N}=160)$ remained in the migraine or migraine plus TTH category seven months later (95\%-CI [69.88-83.17]) as compared to only $44.71 \%$ (95\%-CI [33.91-53.89]) of the adolescents initially in the probable migraine group $(\mathrm{N}=85)$ (Figure 2).

The mean of number of days with headache in the preceding three months was 8.74 and only $13.71 \%$ reported mild headache. $5.14 \%$ of the students reported taking analgesics for every headache attack, 11.95\% in 
Table 1 Shifting of headache diagnoses from baseline to follow-up in 783 students with headache

\begin{tabular}{cccccc}
\hline & \multicolumn{5}{c}{ Follow-up } \\
\cline { 3 - 6 } & & Migraine & TTH & \multicolumn{4}{c}{ Mig + TTH } & MiscH \\
\% & \multicolumn{5}{c}{ (N) } \\
\cline { 3 - 6 } Baseline & Migraine & 54.29 & 21.22 & 11.43 & 13.06 \\
& $\mathbf{N}=\mathbf{2 4 5}$ & $(133)$ & $(52)$ & $(28)$ & $(32)$ \\
TTH & 15.51 & 50.95 & 11.71 & 21.84 \\
$\mathbf{N}=\mathbf{3 1 6}$ & $(49)$ & $(161)$ & $(37)$ & $(69)$ \\
Mig + TTH & 36.84 & 26.32 & 22.11 & 14.74 \\
$\mathbf{N}=\mathbf{9 5}$ & $(35)$ & $(25)$ & $(21)$ & $(14)$ \\
$\mathbf{M i s c H}$ & 13.39 & 33.07 & 8.66 & 44.88 \\
$\mathbf{N}=\mathbf{1 2 7}$ & $(17)$ & $(42)$ & $(11)$ & $(57)$ \\
\hline
\end{tabular}

nearly every headache attack, $14.78 \%$ sometimes, $22.88 \%$ rarely and $45.24 \%$ never. Possible medication overuse headache appeared possible in $1.28 \%$ of the adolescents at baseline (at least 15 headache attacks per month for the last three months treated with analgesics).

\section{Discussion}

After a follow-up period of seven months, the stability of a confirmed diagnosis of either migraine or TTH in adolescents was high. Stability of diagnosis was lower for those with a probable diagnosis initially.
Compared to other studies, the diagnosis stability found in this study for both migraine and TTH was comparatively low [4-7]. A potential explanation for this might be a different proportion of individuals with confirmed versus probable migraine in different study populations. While most studies do not address this issue, two studies point to a higher stability of the confirmed diagnosis of migraine $[4,7]$. Our data are the first to show a significantly better diagnosis stability of confirmed migraine diagnosis as indicated by clearly disjunctive $95 \%$ confidence intervals.

The target of many headache intervention programs is to avoid risk factors for headache. Some of these risk factors are specific for certain headache types; for example, smoking and coffee consumption are strongly associated with migraine but not with TTH [13-16]. Thus in order to undertake headache-specific interventions, it is important to first understand the stability of a headache diagnosis.

Although the questionnaire used was not entirely validated parts of it had been $[17,18]$. The extension of the validated questionnaire was necessary in order to include all questions required to use the criteria of the ICHD-3 beta. Similar extensions have previously been used in other studies $[11,19,20]$. These extended versions have been shown to produce reasonably prevalence of headache and headache types. And although diagnosis of headache in children and adolescents is more difficult than in adults, good sensitivities of between $60-70 \%$ for

\section{Baseline}

Follow-up

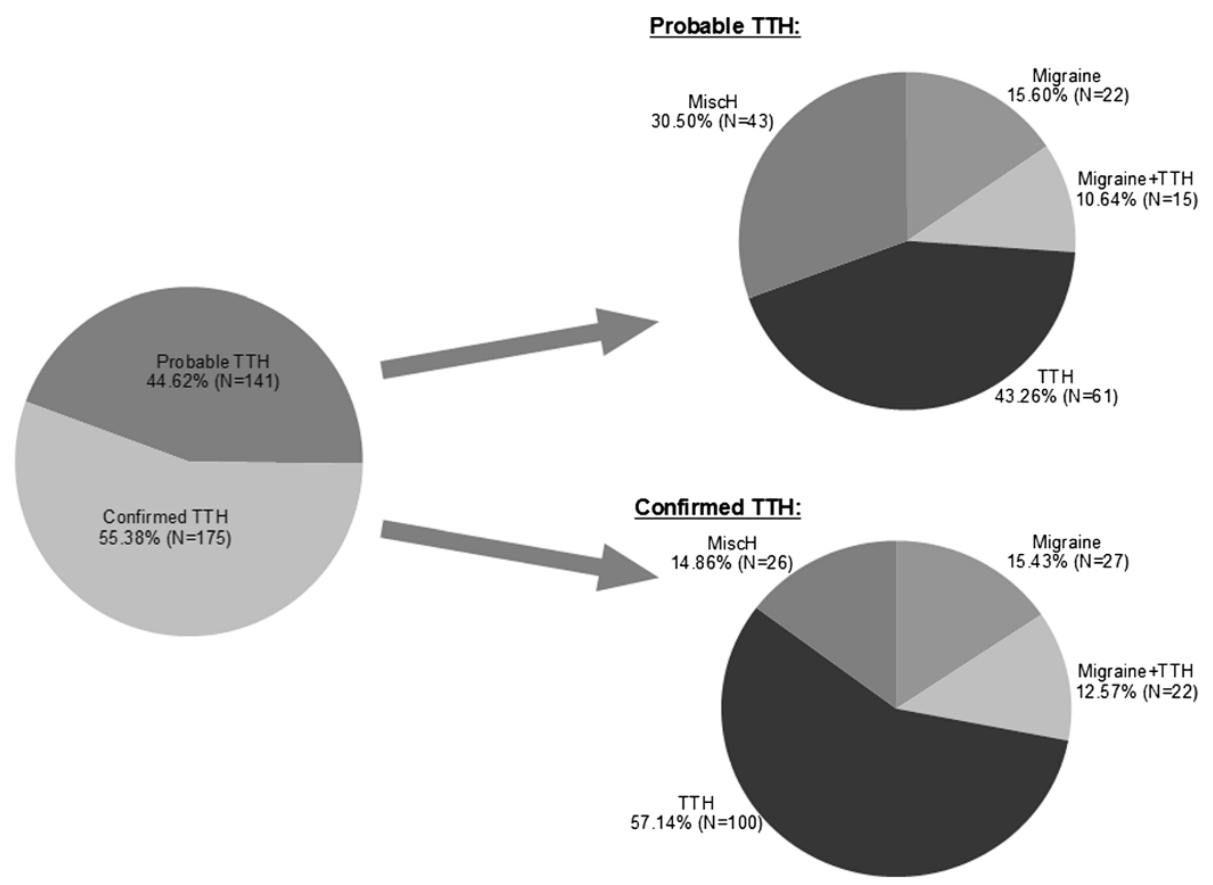

Figure 1 Development of headache type in $316 \mathrm{TTH}$ patients after a follow-up of 7 months. 


\section{Baseline}

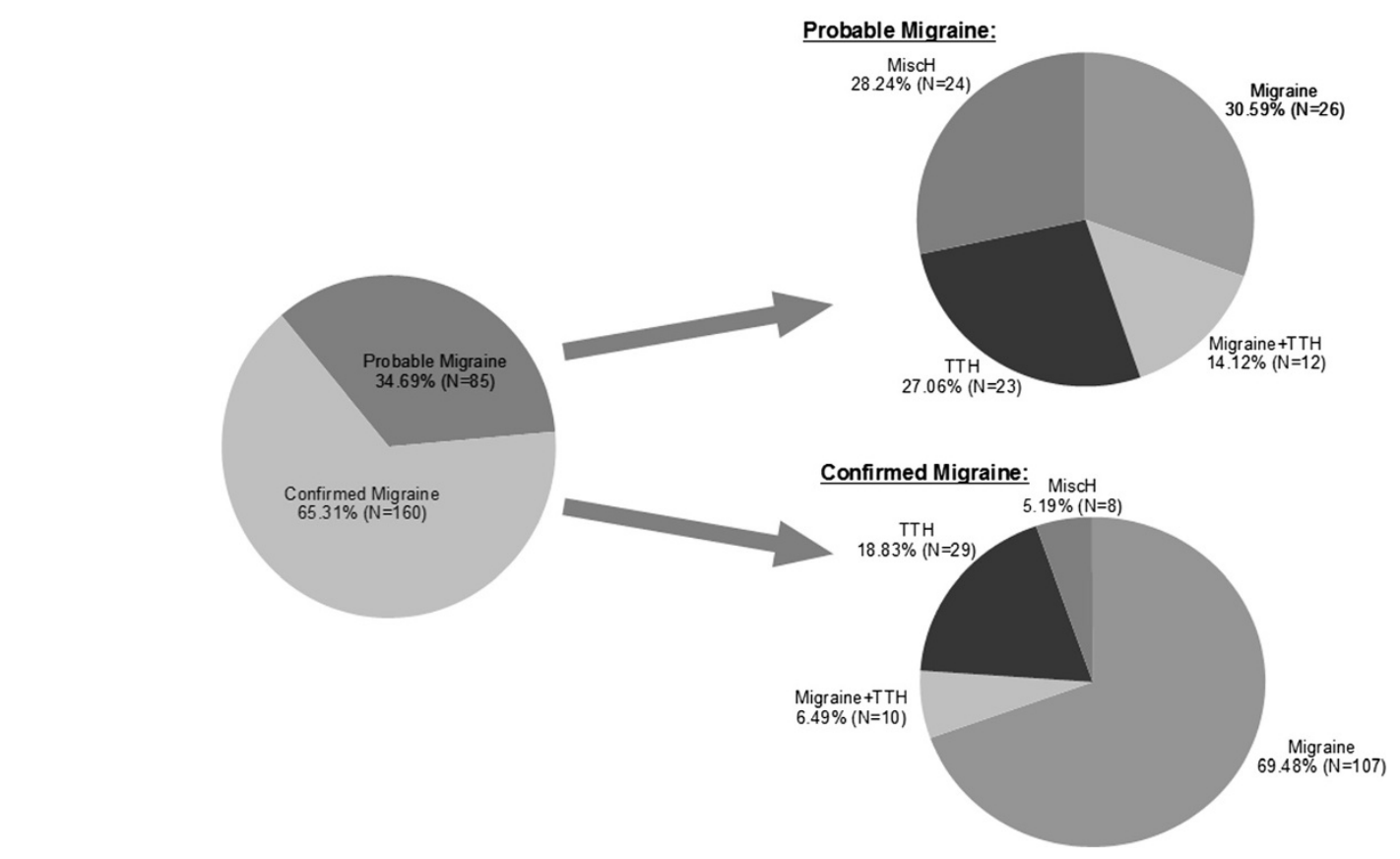

Follow-up

Figure 2 Development of headache type in 245 migraine patients after a follow-up of 7 months. similar screening questionnaires according to the ICHD-II were found (with the diagnosis of a headache specialist as gold standard) [21,22]. A high specificity (100\%) for ICHD-II diagnosis of migraine as compared to an extensive interview by a headache specialist has been identified [21]. The Cohen's kappa coefficient was 0.66 denoting a good level of agreement between both analyses.

Our study provides valid prevalence estimates for different headache types in grammar school students in Munich since all students in class at the first assessment answered the questionnaire. Follow-up was not related to type of headache, and thus precludes attrition bias. Potential causes for poor stability of diagnosis could be [2]: focus on only one headache type in the questionnaires thus ignoring potential occurrence of two headache types in one person (which is potentially relevant if the prevailing symptoms change over time); emergence of a new headache type (which is possible in our data); or poor reliability (unlikely as fair reliability of the questionnaire was recently demonstrated in structured interviews based on the International Headache Society (IHS) classification [23]). It is possible that the comparability of a self-administered questionnaire and a semi-structured interview may be limited, but from other fields of research there is some indication that the results of a self-administered questionnaire and structured interview might differ only marginally [24].
Although the questionnaire was self-administered, the students had the opportunity to ask questions for clarification within the classroom setting. A physician or a psychologist knowledgeable about the content of the questionnaire was present in the classroom.

A limitation of the study might be that we had no opportunity to identify secondary headache types. We are less concerned about this, however, since the average number of headache episodes in the last three months was only 8.7 , suggesting that mild episodic secondary headache is unlikely to account for bias and only $14 \%$ of the study population reported having mild headache. Medication overuse headache $(\mathrm{MoH})$ was not assessed in the questionnaire, but according to the review of Stovner and Andree only 1-2\% of the European population suffer from $\mathrm{MoH}$ [25]. In our baseline data, only $1.28 \%$ of the adolescents report to have at least 15 headache attacks per month for the last 3 months and take analgesics for every or nearly every headache attack, therefore few of our headache cases are likely to be related to $\mathrm{MoH}$.

A further limitation of our analysis might be the use of data from both arms of an intervention study for comparison of baseline and follow-up data. Since no significant difference could be found regarding diagnosis stability between control and intervention group such bias appears unlikely. 


\section{Conclusion}

Fair stability of confirmed migraine and confirmed tensiontype headache was found in our study, whereas stability of probable migraine diagnosis and probable TTH diagnosis proved considerably lower.

\section{Abbreviations}

TTH: Tension-type headache; Mig + TTH: Migraine plus tension-type headache; MiscH: Miscellaneous headache; $\mathrm{MoH}$ : Medication overuse headache; OR: Odds ratio; Cl: Confidence interval; ICHD: International classification of headache disorder

\section{Competing interests}

The authors declare that they have no competing interests.

\section{Authors' contributions}

LA carried out the initial analyses, drafted the initial manuscript, and approved the final manuscript as submitted. FH, AS, MNL, RvK conceptualized and designed the study, revised the manuscript and approved the final manuscript as submitted.

\section{Acknowledgements}

We would like to thank all participating students and teachers and the Bavarian ministry of education for giving the opportunity to perform this study. We also thank the DMKG (German Headache Society), which supported the study by an unrestricted fund.

\section{Author details}

'Division of Epidemiology, Institute of Social Paediatrics and Adolescent Medicine, Ludwig-Maximilians-University Munich, Haydnstr. 5, 80337 Munich, Germany. ²Department of Neurology, Ludwig-Maximilians-University Munich, Munich, Germany. ${ }^{3}$ Department of Paediatric Neurology and Developmental Medicine, Dr. von Hauner Children's Hospital, Ludwig-Maximilians-University Munich, Munich, Germany.

Received: 19 February 2014 Accepted: 30 April 2014 Published: 10 June 2014

\section{References}

1. Wöber-Bingöl C (2013) Epidemiology of migraine and headache in children and adolescents. Curr Pain Headache Rep 17(6):341

2. Beghi E (2004) Methodology of studies on the epidemiology of headache. Neurol Sci Off J Ital Neurol Soc Ital Soc Clin Neurophysiol 25(Suppl 3):S70-S73

3. Van Gessel H, Gassmann J, Kröner Herwig B (2011) Children in pain: recurrent back pain, abdominal pain, and headache in children and adolescents in a four-year-period. J Pediatr 158(6):977-983. e1-2

4. Kienbacher C, Wöber C, Zesch HE, Hafferl-Gattermayer A, Posch M, Karwautz A, Zormann A, Berger G, Zebenholzer K, Konrad A, Wöber-Bingöl C (2006) Clinical features, classification and prognosis of migraine and tension-type headache in children and adolescents: a long-term follow-up study. Cephalalgia Int J Headache 26(7):820-830

5. Zebenholzer K, Wöber C, Kienbacher C, Wöber-Bingöl C (2000) Migrainous disorder and headache of the tension-type not fulfilling the criteria: a follow-up study in children and adolescents. Cephalalgia Int J Headache 20(7):611-616

6. Guidetti V, Galli F (1998) Evolution of headache in childhood and adolescence: an 8-year follow-up. Cephalalgia Int J Headache 18(7):449-454

7. Camarda R, Monastero R, Santangelo G, Raimondo D, Puma D, Pipia C, Camarda LKC, Camarda C, Raieli V (2002) Migraine headaches in adolescents: a five-year follow-up study. Headache 42(10):1000-1005

8. Metsähonkala L, Sillanpää M, Tuominen J (1997) Outcome of early school-age migraine. Cephalalgia Int J Headache 17(6):662-665

9. Ozge A, Sasmaz T, Cakmak SE, Kaleagasi H, Siva A (2010) Epidemiological-based childhood headache natural history study: after an interval of six years. Cephalalgia Int J Headache 30(6):703-712

10. Gassmann J, Morris L, Heinrich M, Kröner-Herwig B (2008) One-year course of paediatric headache in children and adolescents aged 8-15 years. Cephalalgia Int J Headache 28(11):1154-1162
11. Fendrich K, Vennemann M, Pfaffenrath V, Evers S, May A, Berger K, Hoffmann W (2007) Headache prevalence among adolescents-the German DMKG headache study. Cephalalgia Int J Headache 27(4):347-354

12. Headache Classification Committee of the International Headache Society (IHS) (2013) The International Classification of Headache Disorders, 3rd edition (beta version). Cephalalgia Int J Headache 33(9):629-808

13. Milde-Busch A, Blaschek A, Borggrafe I, Heinen F, Straube A, von Kries R (2010) Associations of diet and lifestyle with headache in high-school students: results from a cross-sectional study. Headache 50(7):1104-1114

14. Winter AC, Hoffmann W, Meisinger C, Evers S, Vennemann M, Pfaffenrath V, Fendrich K, Baumeister SE, Kurth T, Berger K (2011) Association between lifestyle factors and headache. J Headache Pain 12(2):147-155

15. Schramm SH, Obermann M, Katsarava Z, Diener H-C, Moebus S, Yoon M-S (2013) Epidemiological profiles of patients with chronic migraine and chronic tension-type headache. J Headache Pain 14(1):40

16. Hagen K, Thoresen K, Stovner LJ, Zwart J-A (2009) High dietary caffeine consumption is associated with a modest increase in headache prevalence: results from the Head-HUNT Study. J Headache Pain 10(3):153-159

17. Roth-Isigkeit A, Raspe HH, Stöven H, Thyen U, Schmucker P (2003) Pain in children and adolescents-results of an exploratory epidemiological study. Schmerz Berl Ger 17(3):171-178

18. Roth-Isigkeit A, Thyen U, Raspe HH, Stöven H, Schmucker P (1992) (2004) Reports of pain among German children and adolescents: an epidemiological study. Acta Paediatr Oslo Nor 93(2):258-263

19. Milde-Busch A, Heinrich S, Thomas S, Kühnlein A, Radon K, Straube A, Bayer O, von Kries R (2010) Quality of life in adolescents with headache: results from a population-based survey. Cephalalgia Int J Headache 30(6):713-721

20. Milde-Busch A, Blaschek A, Heinen F, Borggrafe I, Koerte I, Straube A, Schankin C, von Kries R (2011) Associations between stress and migraine and tension-type headache: results from a school-based study in adolescents from grammar schools in Germany. Cephalalgia 31(7):774-785

21. Valentinis L, Valent F, Mucchiut M, Barbone F, Bergonzi P, Zanchin G (2009) Migraine in adolescents: validation of a screening questionnaire. Headache 49(2):202-211

22. Hershey AD, Winner P, Kabbouche MA, Gladstein J, Yonker M, Lewis D, Pearlman E, Linder SL, Rothner AD, Powers SW (2005) Use of the ICHD-II criteria in the diagnosis of pediatric migraine. Headache 45(10):1288-1297

23. Granella F, D'Alessandro R, Manzoni GC, Cerbo R, Colucci D'Amato C, Pini LA, Savi L, Zanferrari C, Nappi G (1994) International Headache Society classification: interobserver reliability in the diagnosis of primary headaches. Cephalalgia Int J Headache 14(1):16-20

24. Steffen MW, Murad MH, Hays JT, Newcomb RD, Molella RG, Cha SS, Hagen PT (2014) Self-Report of Tobacco Use Status: Comparison of Paper-Based Questionnaire, Online Questionnaire, and Direct Face-to-Face Interview-Implications for Meaningful Use. Popul Health Manag. doi:10.1089/pop.2013.005

25. Stovner LJ, Andree C (2010) Prevalence of headache in Europe: a review for the Eurolight project. J Headache Pain 11(4):289-299

\section{doi:10.1186/1129-2377-15-36}

Cite this article as: Albers et al:: High diagnostic stability of confirmed migraine and confirmed tension-type headache according to the ICHD-3 beta in adolescents. The Journal of Headache and Pain 2014 15:36.

\section{Submit your manuscript to a SpringerOpen ${ }^{\odot}$ journal and benefit from:}

- Convenient online submission

- Rigorous peer review

- Immediate publication on acceptance

- Open access: articles freely available online

- High visibility within the field

- Retaining the copyright to your article

Submit your next manuscript at $>$ springeropen.com 